\title{
Pediatric neurocysticercosis: current challenges and future prospects
}

This article was published in the following Dove Press journal:

Pediatric Health, Medicine and Therapeutics

8 March 2016

Number of times this article has been viewed

Pratibha Singhi

Arushi Gahlot Saini

Department of Pediatrics, Pediatric Neurology and Neurodevelopment Unit, Advanced Pediatrics Centre, Post Graduate Institute of Medical Education and Research, Chandigarh, India
Correspondence: Pratibha Singhi Department of Pediatrics, Pediatric Neurology and Neurodevelopment Unit, Advanced Pediatric Centre,

Post Graduate Institute of Medical Education and Research, Madhya Marg, Sector-12, Chandigarh 1600I2, India

Tel +9l I72 275 530I/274 6699

Fax +9I I72 $274440 I / 2747099$

Email doctorpratibhasinghi@gmail.com
Abstract: Neurocysticercosis (NCC) is an acquired infection of the nervous system caused by encysted larvae of Taenia solium. It is a major cause of epilepsy in the tropics and the commonest cause of focal seizures in North Indian children. T. solium teniasis-cysticercosis is considered a parasitic "Neglected Tropical Diseases" endemic throughout Southeast Asia. NCC in children has pleomorphic manifestations depending on the location, number and viability of the cysts, and host response. Even with advancing knowledge of the disease manifestations, many aspects related to diagnosis and treatment, particularly in children, still remain controversial and pose challenges to clinical practice. There is no gold standard test to diagnose NCC and the management recommendations are still emerging. This review provides an overview of diagnosis of NCC in children and its management with special focus on current challenges and future prospects.

Keywords: neurocysticercosis, children, epilepsy, ring enhancing lesions, pigs

\section{Introduction}

Neurocysticercosis (NCC) is an acquired infection of the nervous system caused by encysted larvae of Taenia solium. It is the most common helminth infection of the nervous system in humans, a major cause of epilepsy in the tropics, and the commonest cause of focal seizures in North Indian children. ${ }^{1,2}$ T. solium teniasis-cysticercosis has been identified as parasitic "Neglected Tropical Diseases" endemic throughout Southeast Asia by World Health Organization. ${ }^{3}$ Although predominantly a disease of the developing countries, it is also seen in many developed countries due to the immigrant population from endemic areas and increasing international travel. NCC in children has pleomorphic manifestations depending on the location, number and viability of the cysts, and host response. With advancing knowledge of the disease manifestations, many aspects related to the diagnosis and treatment in children still remain poorly understood and pose challenges to clinical practice. This review provides an overview of diagnosis of NCC in children and its management with special focus on current challenges and future prospects.

\section{Disease burden: the first challenge}

The limitations in the estimation of true disease burden include lack of systematic population-based studies, community surveillance programs, or case notification systems; lack of gold standard tests to either screen the carrier population or to detect asymptomatic cysticercosis; limited role of serology in endemic areas where individuals may be seropositive even without harboring NCC; and nonfeasibility of computed 
tomography (CT) brain scans to screen asymptomatic population. In addition, the available studies have used different methodologies and highly selected populations, and there is an absence of basic or uniform definitions. Therefore, data from these studies cannot be generalized.

NCC is endemic in most developing countries, particularly Latin America, the Indian subcontinent, Southeast Asian region, and sub-Saharan Africa. Increasing globalization and travel has increased the incidence of NCC in many nonendemic countries including USA, UK, and Australia. ${ }^{4,5}$ A recent systematic review on cysticercosis and T. solium teniasis in Europe showed an increasing trend; nearly $75 \%$ of imported cases were diagnosed in migrants and $18 \%$ in European travelers. ${ }^{6}$ The data from developing countries are more variable. Prevalence of human teniasis varies from $2 \%-10 \%$ in rural Africa, $24 \%-37 \%$ in Peru, and 6.5\%-28\% in India. ${ }^{7-14}$ Using CT scans, $10 \%-20 \%$ of the general population showed residual brain parenchymal calcifications in endemic areas of Peru and Honduras. ${ }^{9,15}$ Based on slaughterhouse surveys, cysticercosis also appears to be widespread (7\%-26\%) among swine in India. ${ }^{16,17}$

Amongst the symptomatic population, greater than 5 million cases of preventable epilepsy worldwide are caused by NCC. ${ }^{18}$ In a recent systematic review, the pooled estimate of the proportion of NCC among people with epilepsy was as high as 29\% from Latin America, sub-Saharan Africa, and Southeast Asia. ${ }^{19}$ A meta-analysis on the association between cysticercosis and epilepsy in Africa showed an overall significant association with a common odds ratio (OR) of 3.4. ${ }^{20}$ Amongst patients with active epilepsy in North India, 25\% of the cases tested positive for antibodies against T. solium. ${ }^{21}$ The point prevalence of 4.5 per 1,000 population has been noted in rural Northwest India. ${ }^{22}$ Among children with partial seizures, NCC accounted for $>50 \%$ cases in hospital-based series from India. ${ }^{23}$

\section{Understanding the etiopathogenesis: a basic step toward prevention}

The life cycle of T. solium (adult tapeworm) involves two hosts, humans and pigs. Humans are the definitive hosts and acquire intestinal infection (teniasis) from pigs, the intermediate hosts, by ingestion of undercooked pork infected with live cysticerci (encysted larvae) (Figure 1). In addition to pigs, dogs may also act as intermediate hosts for T. solium and consumption of dog meat in certain Asian countries such as People's Republic of China, Korea,

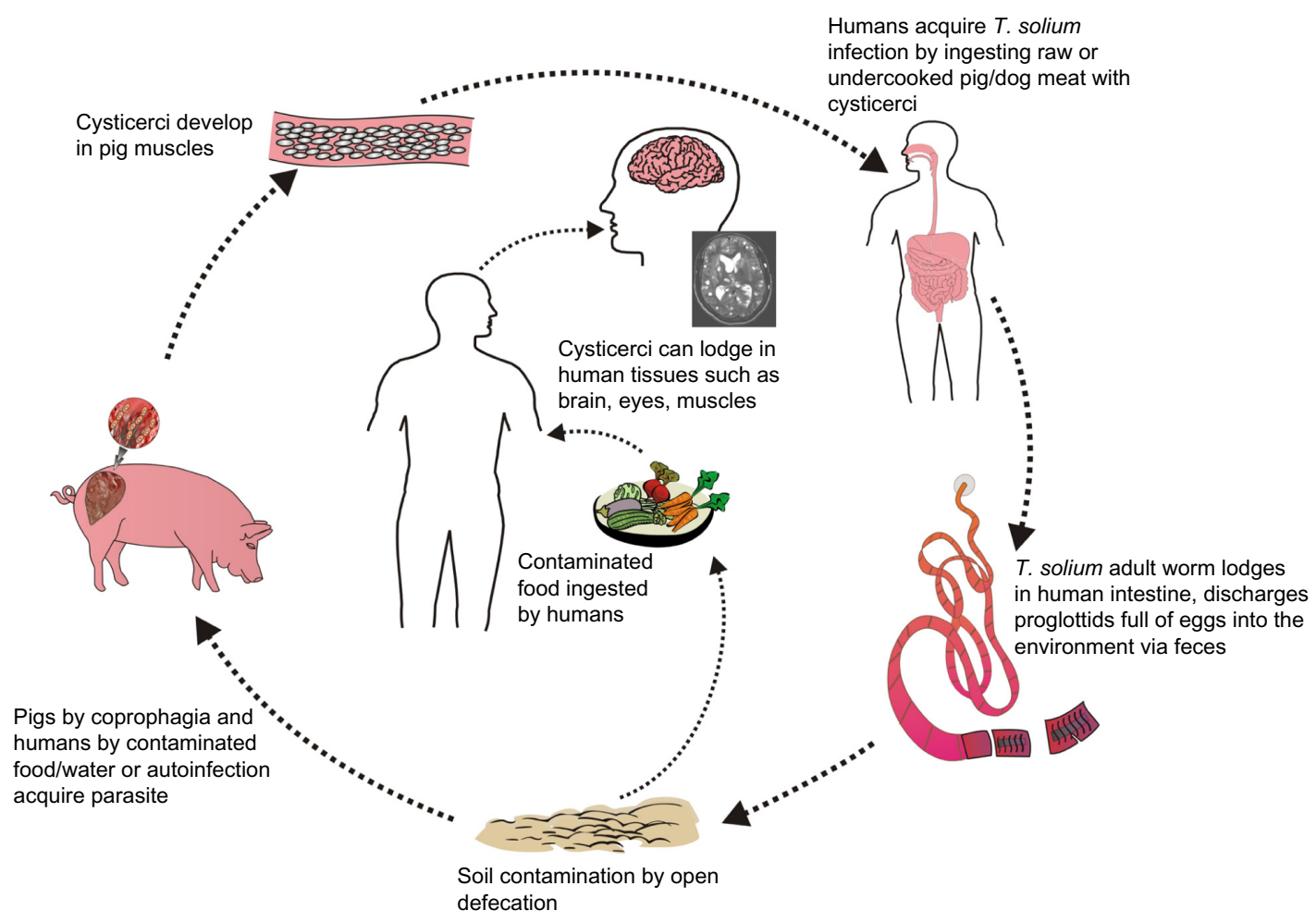

Figure I Life cycle of NCC.

Notes: The life cycle of Taenia solium (adult tapeworm) involves two hosts: humans and pigs. Humans are the definitive hosts and acquire intestinal infection (teniasis) from pigs, the intermediate hosts, by ingestion of undercooked pork infected with live cysticerci (encysted larvae). Humans acquire cysticercosis via consumption of food or water contaminated with T. solium eggs or by autoinfection. Neurocysticercosis (NCC) results when the larval stages lodge in the brain. 
Vietnam, Indonesia, and Thailand could, therefore, put individuals at risk for cysticercosis. ${ }^{24} \mathrm{NCC}$ is acquired feco-orally by consuming food or water contaminated by eggs either accidently or via infected food handlers (carriers of tapeworm), or rarely by autoingestion. This person-to-person transmission of cysticercosis assumes importance in the Indian subcontinent where majority of the population is vegetarian, in children handled by infected babysitters, and in the orthodox Jewish communities of USA where domestic Latin American employees are carriers. ${ }^{25,26}$ The repetitive feco-oral autoinfections amongst T. solium carriers have been shown to result in implantation of hundreds of parasites in the nervous system without triggering a severe inflammatory reaction or encephalitis symptoms, due to poorly understood immunosuppressive mechanisms. ${ }^{27}$

Most cysts are located in the brain parenchyma and therefore cause seizures. The cysts pass through a sequence of four morphological stages: vesicular, colloidal, granular nodular, and nodular calcified stage. ${ }^{28}$ In the Indian subcontinent, neuroimaging of symptomatic cases of NCC usually shows colloidal or granular nodular stages of the cyst, whereas in Latin American countries vesicular stages are often seen. Although calcified cysticerci are thought to represent dead and inactive cysts, these are not totally inert and may intermittently release parasite antigens that result in inflammatory edema and symptoms. ${ }^{26,29}$ However, it remains debatable whether this edema is the cause or the consequence of seizures. ${ }^{30}$ Even the perilesional gliosis or recently described "associated hippocampal sclerosis" in these patients may trigger recurrent seizures if antiepileptic drugs (AEDs) are withdrawn prematurely. ${ }^{31-33}$ When the cysts are extraparenchymal (ventricles, subarachnoid space, cisterns), they tend to grow irregularly according to the available space and elicit a strong inflammatory response. They may assume enormous size without scolices (racemose NCC) that have peculiar appearances and mass effects. Intraventricular cysts may cause acute hydrocephalus due to direct or inflammatory obstruction.

\section{Challenges in diagnosis}

The diagnosis of teniasis is difficult in endemic settings due to the following reasons: ${ }^{34}$

- Most of the tapeworm carriers are asymptomatic and therefore are not investigated.

- T. solium eggs are found in the feces only when the eggs have been discharged from gravid proglottids, which may occur infrequently (only once or twice per day).
- Morphological differentiation of T. solium eggs from the eggs of other less pathogenic tapeworms is difficult in resource-poor areas where advanced molecular tests for species differentiation are not available.

- Low sensitivity of tests on live pigs and poor inspection systems at slaughterhouses in most developing countries make identification of the infected reservoir population of the pigs difficult.

Although diagnostic criteria for incorporating clinical, radiological, immunological, and epidemiological data have been proposed for NCC, their applicability is more in research rather than routine day-to-day clinical practice, especially in resource-constraint settings. ${ }^{35,36}$ The clinical manifestations in children are pleomorphic depending on the burden of cysts, their location, and size. NCC should be suspected clinically in any normally developing child with sudden-onset seizures, headache, vomiting, or focal motor deficits where there is no other suggestion of an underlying neurological disorder. However, in endemic areas, physicians should keep their eyes and minds open to the atypical presentations of NCC such as communicating hydrocephalus, vasculitis, stroke, dorsal midbrain syndrome, ptosis, amaurosis fugax, dystonia, neurocognitive deficits, and psychiatric presentations. ${ }^{37,38}$ Due to the lack of specific neurological symptoms, diagnosis on clinical grounds alone is impossible and needs to be substantiated with neuroimaging and serology.

\section{Imaging characteristics of typical parenchymal neurocysticercosis}

The imaging characteristics of typical parenchymal $\mathrm{NCC}^{39-41}$ vary according to the pathological staging of the cyst. ${ }^{28}$

- Vesicular stage: characterized by small cyst with fluid similar to cerebrospinal fluid (CSF), thin wall, and an eccentrically located scolex, showing no contrast enhancement of the cyst's wall and no surrounding tissue edema.

- Colloidal vesicular stage: density and signal intensity of the cystic fluid is different from that of CSF, and shows a thicker cyst wall, ill-defined shrunken scolex, and ringlike enhancement with perilesional edema.

- Granular nodular stage: characterized by small enhancing cyst or nodule, with mild surrounding edema and little mass effect.

- Nodular calcified stage: characterized by small calcified nodule with no edema and is best seen on CT.

In the Indian subcontinent, the commonest CT finding is a single, small $(<20 \mathrm{~mm})$, low-density lesion with ring or disc enhancement termed as single small enhancing computed tomographic lesion (SSECTL) representing a degenerating 
cyst with associated mild-to-moderate perilesional edema and bright, hyperdense, eccentric scolex pathognomonic for NCC (Figure 2A and B). ${ }^{42}$ It is also termed single cysticercus granuloma. Numerous cysts of varying stages may give rise to a typical "starry-sky" appearance. CT scan may miss posterior fossa lesions. Magnetic resonance imaging (MRI) is more sensitive for detection of the scolex (Figure 3A-C) and extraparenchymal NCC, though tiny calcified lesions (Figure 2C) may be missed on routine MRI scans and are better visualized on susceptibility-weighted images.

\section{Diagnosing a ring enhancing lesion without a visible scolex}

If the scolex is not seen, the most important differential diagnosis in most resource-limited countries is tuberculoma, followed by other uncommon causes in children such as micro-abscesses, toxoplasmosis, fungal lesions, low-grade astrocytoma, and cystic cerebral metastasis..$^{43}$ The following imaging features favor a tuberculoma: size of CT lesion $>20 \mathrm{~mm}$, location at the base of the brain/posterior fossa (NCC lesions are often seen at the gray-white matter junction), lobulated irregular shape, marked edema causing midline shift, presence of raised intracranial pressure, progressive focal neurodeficits, presence of a lactate peak, and choline/creatine ratio $>1$ on MR spectroscopy. ${ }^{35-37,44,45}$

Fluid-attenuated inversion recovery (FLAIR) MRI (Figure 3C) and diffusion-weighted images frequently improve the visualization of the scolex. ${ }^{46}$ Higher apparent diffusion coefficient is seen in the core of cysticercal cysts as compared to tuberculomas or tuberculous abscesses. ${ }^{47}$ Additional sophisticated and advanced imaging techniques may be needed. Proton MR spectroscopy helps in the identification of inflammatory granulomas - a lipid peak suggests a tuberculoma whereas low levels of metabolites with poor signal/ noise ratio suggest NCC. ${ }^{44}$ Magnetization transfer images and magnetization transfer ratio help in the identification of perilesional gliosis and lesions not visible on routine MRI. Persistent perilesional gliosis identified by magnetization transfer images is associated with refractory epilepsy in patients with NCC. ${ }^{48}$ FLAIR sequence with $100 \%$ supplemental $\mathrm{O}_{2}$ can increase the signal intensity of CSF, while three-dimensional constructive interference in steady state (CISS) is a heavily three-dimensional T2-weighted high-resolution cistern imaging sequence. Both methods can help in differentiating cyst lesion from $\mathrm{CSF}^{49}$ Also, three-dimensional MRI sequences have been found useful to diagnose basal subarachnoid NCC which is difficult to diagnose with classical MRI. ${ }^{50}$

\section{Immunodiagnostic tests}

An ideal immunodiagnostic test for NCC should be nonexpensive, feasible, easily available, point-of-care with good sensitivity and specificity not only for multiple lesions but also for single lesions. The role of these tests is currently as adjunct to imaging techniques. Numerous tests are available using different parasitic antigens (total metacestode soluble antigens, metacestode membrane or scolex soluble extracts, and semipurified proteins), but there is as yet no ideal immunodiagnostic test particularly for single-lesion NCC.

\section{Antigen detection tests}

Several antigen detection methods have been used; however, none is ideal. Use of crude antigenic preparations has shown only moderate sensitivity and specificity. Semipurified/purified antigens are associated with better yield but are complex

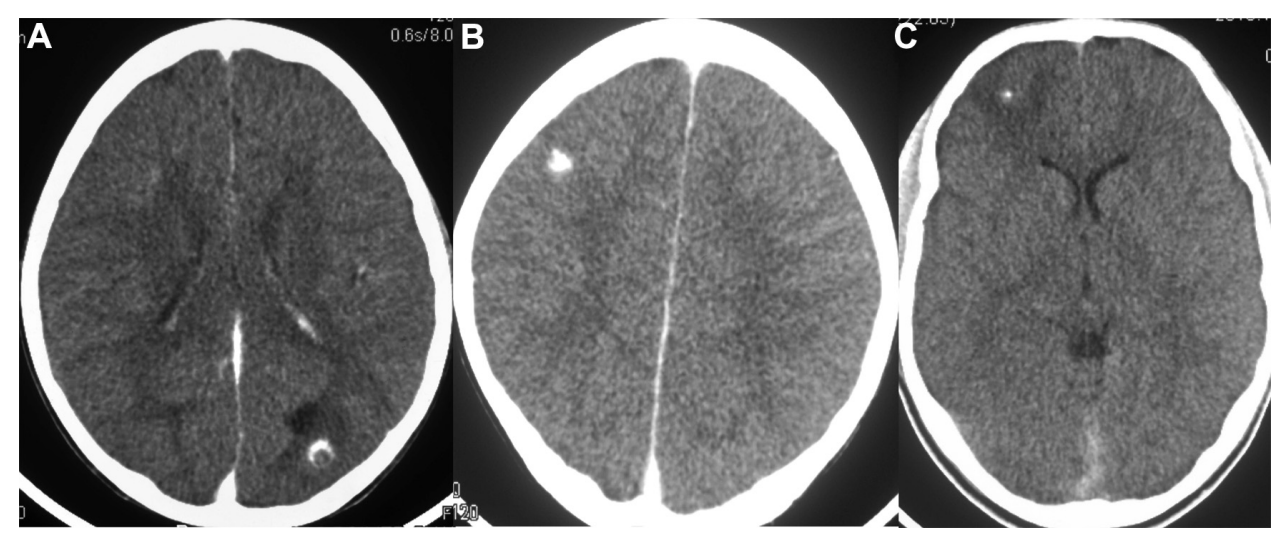

Figure 2 Brain CT scan.

Notes: Contrast-enhanced axial section showing a single, small $(<20 \mathrm{~mm})$, hypodensity lesion with (A) ring and (B) disc enhancement, termed as single small enhancing computed tomographic lesion (SSECTL) representing a degenerating cyst with associated mild-to-moderate perilesional edema and bright, hyperdense, eccentric scolex pathognomonic for NCC. (C) Small calcified nodule with mild perilesional edema.

Abbreviations: CT, computed tomography; NCC, neurocysticercosis. 


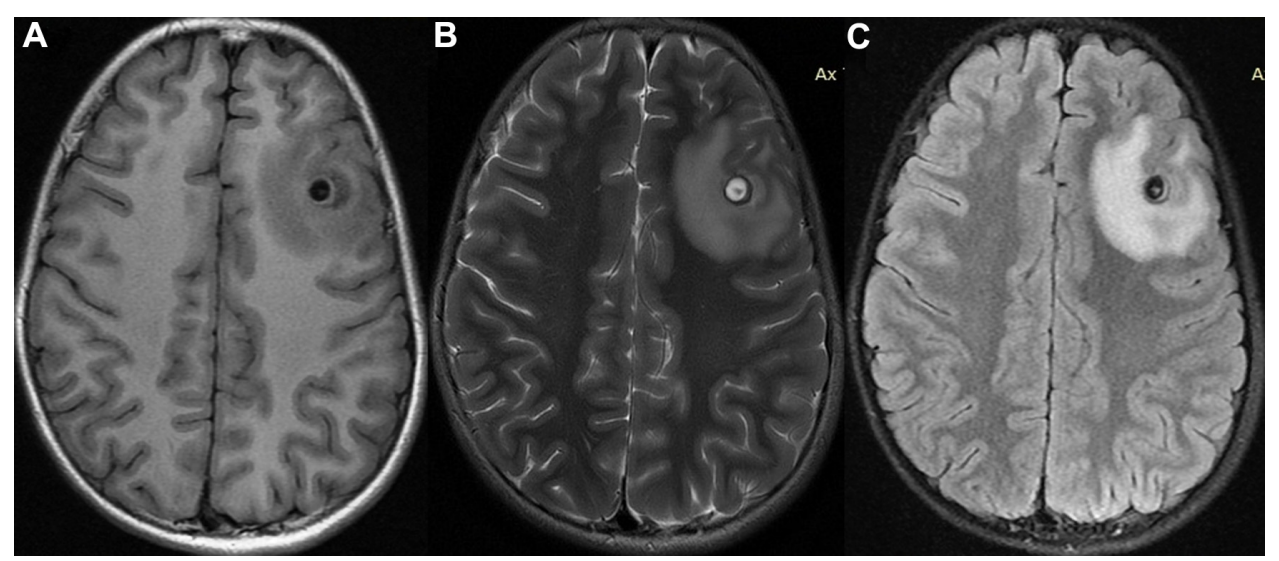

Figure 3 Brain MRI scans.

Notes: (A) Noncontrast axial TI-weighted scan showing small, circular cyst with cerebrospinal-like fluid and a small eccentric scolex seen as intramural nodule, isointense rim suggestive of cyst wall, and hypointense areas surrounding the cyst suggestive of perilesional edema. (B) T2-weighted axial scan showing the same cyst with cerebrospinal-isointense fluid, intramural nodule, hypointense rim suggestive of cyst wall, and hyperintense perilesional edema. (C) FLAIR axial section showing the same hypointense cyst with hyperintense scolex, thin cyst wall, and hyperintense perilesional edema. The presence of scolex is pathognomonic of neurocysticercosis. Abbreviations: MRI, magnetic resonance imaging; FLAIR, fluid-attenuated inversion recovery.

and need technical expertise. Use of excretory antigens such as $67 \mathrm{kDa}, 43 \mathrm{kDa}$, and $32 \mathrm{kDa}$ peptides rather than somatic antigens has been shown to be superior for vesicular stages of the parasite in both CSF and serum. ${ }^{51-53}$ Traditionally, serum and CSF samples have been used but recently noninvasive samples such as urine, saliva, and even tears in ophthalmic cysticercosis are being investigated. A comparative study of antigenic peptides in three different body fluids (CSF, serum, and urine) obtained from confirmed NCC cases and control subjects found that only lower molecular weight antigenic peptides were found in urine, whereas higher molecular weight antigenic peptides were found in serum and CSF. ${ }^{54}$ Also, somatic as well as excretory antigens were detected with highest sensitivity in the CSF specimen followed by serum and urine. The highest efficacy overall (90\%-91\%) for detecting antigens was in the CSF. ${ }^{54}$

\section{Antibody detection tests}

The antibody responses in serum may help in diagnosis as well as evaluation of therapeutic responses in patients with NCC. ${ }^{55}$ Seropositivity depends on the parasite load and on genotypes of T. solium (Asian versus African/Latin American). ${ }^{56} \mathrm{~A}$ positive serological test supports the diagnosis of NCC but a negative test does not exclude it. Low positivity from $17 \%-25 \%$ has been reported in children. ${ }^{2}$

Lentil lectin purified glycoproteins-based enzyme-linked immunoelectrotransfer blot (EITB) assay is currently the best serodiagnostic method. ${ }^{51}$ EITB assay using purified T. solium cysticercal antigens was reported to be highly specific (100\%) and sensitive (98\%) for active multiple and extraparenchymal lesions in hospitalized patients. ${ }^{57}$ However, the sensitivity was lower $(50 \%-70 \%)$ in solitary cysticercus granuloma and calcified NCC. Even enzyme-linked immunosorbent assay (ELISA) and dot-blot assay in children aged 5-12 years were found to be more sensitive in multiple lesions (100\%) as compared to single lesions $(87 \%){ }^{58}$

Our study showed that the excretory antigen is a better diagnostic antigen than crude soluble extract or lower molecular mass antigens for detection of antibody in NCC children. Also, this study was the first to demonstrate that serum is the better diagnostic sample than urine. ${ }^{53}$ EITB has similar sensitivity for serum and CSF samples while ELISA has better sensitivity with CSF samples. ${ }^{59}$ Due to tedious purification process, an immunoblot assay (recombinant EITB) has been developed with 99\% sensitivity and specificity for diagnosing NCC. ${ }^{60}$ False-positive serological tests may be seen in extraneural cysticercosis, other cestode and helminth infections, and up to 1 year after antiparasitic therapy in neural cysts. ${ }^{61}$ Immunoblot-based detection of antibodies against 26 and $8 \mathrm{kDa}$ antigens approaches nearly $100 \%$ specificity but is less sensitive than EITB. ${ }^{62}$ Lower molecular mass (20-24 kDa) cysticercal antigens for serodiagnosis by ELISA and dot-blot in children has also been evaluated. ${ }^{63}$ Synthetic peptide selected by phage display has also been used for immunodiagnosis of NCC. ${ }^{64}$ Diethylaminoethyl binding fraction from $T$. solium metacestode has been reported to improve the NCC serodiagnosis. ${ }^{65}$ Recently, two magnetic immune-chromatographic tests for serologic detection of teniasis (based on rES33 antigen) and cysticercosis (based on the rT24H antigen) have shown high sensitivity and specificity in cases with $\geq 2$ viable brain cysts. ${ }^{66}$ A multiantigen print immunoassay is also being evaluated as a simple and 
effective tool to compare antibody responses to different cysticercosis and teniasis antigens and for rapid detection of T. solium cases. ${ }^{66}$ A field-friendly rapid lateral flow-based antibody screening test in the serum using bacterial expressed recombinant (r)T24H has shown good sensitivity (96\%) and specificity $(98 \%)$ for NCC. ${ }^{67}$

\section{Polymerase chain reaction (PCR)-based tests}

Parasite DNA detection by PCR has also been used for diagnosis from CSF and fecal samples. ${ }^{68,69}$ Real-time CSF PCR has been shown to confirm the diagnosis of NCC in cases suggested by clinical, imaging, immunologic, and epidemiologic features and has also been shown to detect the persistence of $T$. solium DNA in the brain after many years. ${ }^{70}$ However, the current information on how the DNA is released into the CSF is limited. ${ }^{68}$ Further, the nonfeasibility of utilizing CSF PCR studies in day-to-day practice for diagnosis of NCC limits the widespread utility of these techniques as a lumbar puncture cannot be done on every patient when the diagnosis is fairly established by neuroimaging and immunodiagnostic techniques. Comparison of immunodiagnostic assays (antibody detection by ELISA and EITB, and HP10 antigen detection by ELISA) with PCR-based detection of parasite DNA from CSF suggests the role of PCR primarily in NCC cases not diagnosed by the available radiological or immunological tests. ${ }^{68}$

\section{Other tests}

Peripheral eosinophilia is seen in approximately a third of NCC cases. There is no role of routine testing of CSF samples. In cysticercal meningitis, CSF shows moderate pleocytosis (mostly mononuclear cells, rarely $>300$ cells per $\mathrm{mm}^{3}$ ), increased protein (50-300 $\left.\mathrm{mg} / \mathrm{dL}\right)$, and low glucose levels; hypoglycorrhachia is associated with a poor prognosis. Biopsy of subcutaneous nodules and radiographs of skeletal muscles to detect calcified cysts (cigar-shaped calcification) may provide corroborative evidence in some cases. Quantification of Fas and amino acid neurotransmitters in CSF of patients with NCC did not reveal any specific pattern; ${ }^{71,72}$ these tests have a limited utility.

There is as yet no ideal diagnostic test for single-lesion NCC in particular. Hence, a combination of clinical, radiological (including special imaging sequences), and immunological information may have to be utilized for diagnosis of NCC, especially in cases with single lesions. Author PS has reported performing NCC serology tests for all cases, keeping in mind that a positive serodiagnostic test supports the diagnosis of $\mathrm{NCC}$; however, a negative test does not exclude the diagnosis of NCC. ${ }^{73}$

\section{Challenges in management}

Management of NCC has been debated around several issues: 1) effectiveness of the anti-parasitic drugs in killing the cysts and in improving the clinical outcome (defined as fewer seizures, in patients with intraparenchymal NCC), 2) choice of the optimal cysticidal drug, 3) use of steroids, and 4) optimal duration of AED therapy. Understandably, the benefits of antiparasitic regimens are more evident in patients with multiple viable cysts and less evident in patients with degenerating lesions.

\section{Role of cysticidal drugs}

The efficacy of anthelmintic drugs in live, vesicular, and active parenchymal cysticercosis has been established, but their role in cases with enhancing lesions (considered degenerating cysts) was controversial. ${ }^{74-76}$ In the first double-blind, placebo-controlled trial in 63 children with SSECTL, we found that albendazole ( $15 \mathrm{mg} / \mathrm{kg} /$ day for 4 weeks) administered within 3 months of onset of seizures was associated with a significantly increased and faster disappearance of lesions after 1 month (41\%) (placebo: 16.2\%; $P<0.05)$ and after 3 months (albendazole: $64.5 \%$ versus placebo: $37.5 \%$; $P<0.05)$ as compared with placebo. ${ }^{77}$ Subsequently, several other trials have confirmed this. ${ }^{78-80}$ A recent meta-analysis of 15 randomized trials showed that anthelminthic treatment was associated with significantly increased frequency of granuloma resolution ([OR]: 2.09; 95\% confidence interval [CI]: $1.41-3.00 ; P<0.0003)$ and thus provides support for the use of albendazole (with or without corticosteroids) in the treatment of single granuloma. ${ }^{81}$

Role of cysticidal therapy in control of seizures has been controversial. In the single trial that evaluated the frequency of seizures in patients with vesicular cystic lesions, there was a $67 \%$ reduction in the rate of generalized seizures with treatment $(P=0.006) .{ }^{75}$ The meta-analysis by Otte et al showed that anthelminthic treatment was associated with significantly increased rates of seizure freedom on short term (nonevent OR: 2.45 ; 95\% CI: $1.49-4.03 ; P=0.0004$ ). ${ }^{81}$

On the balance of evidence, cysticidal therapy seems to be effective in reducing the number of lesions and improves rates of seizure freedom. However, its role in improving long-term seizure control needs further larger studies. Commensurate with this, the American Academy of Neurology recommends the use of albendazole and corticosteroids for adults and children with parenchymal 
NCC to reduce both the number of active lesions and longterm seizure frequency. ${ }^{82}$

It is generally agreed that both praziquantel and albendazole are effective in destroying viable cysts. Albendazole is preferred to praziquantel as it is less expensive, better tolerated, has fewer side effects, has better penetration into the subarachnoid space, and its bioavailability increases with coadministration of corticosteroids and remains unaffected by the coadministration of phenytoin or carbamazepine..$^{83,84}$

\section{Dose and duration of cysticidal therapy}

Albendazole is used in a dose of $15 \mathrm{mg} / \mathrm{kg} /$ day in 2-3 divided doses, usually for 28 days particularly in multiple lesions; shorter durations of 8-14 days have also been used. ${ }^{85}$ In a placebo-controlled trial of 1 week versus 4 weeks albendazole therapy in children with 1-3 enhancing lesions, both the regimens were found to be equally effective in resolution of lesions on 3 months CT and seizure control at 1 year. ${ }^{86}$ Praziquantel is used in a dose of $50 \mathrm{mg} / \mathrm{kg} /$ day for 15 days. A single-day praziquantel therapy $(25 \mathrm{mg} / \mathrm{kg} /$ dose every 2 hours $\times 3$ doses) has been reported to be as effective as 7 days treatment with albendazole. ${ }^{87}$ Side effects of praziquantel include abdominal pain, dizziness, headache, and allergic reactions in rare cases.

\section{Role of combination therapy}

A combination therapy of albendazole and praziquantel was found to have no significant difference in the outcome of single-lesion NCC as compared to albendazole alone (although it was associated with a trend to higher resolution). ${ }^{88} \mathrm{~A}$ recent study of 124 patients also demonstrated that the combination of albendazole plus praziquantel increases the parasiticidal effect in patients with multiple NCC without increased side effects. ${ }^{89}$ However, larger trials are needed to establish its efficacy. The question still remains whether combination therapy should be used upfront in all cases or in specific ones. We currently use monotherapy with albendazole in all children as first-line treatment, and praziquantel is added if there are persistent active lesions on neuroimaging.

\section{When not to use cysticidal therapy}

Cysticidal therapy at the outset is contraindicated in children with markedly elevated intracranial pressure and ophthalmic cysticercosis due to the risk of inducing an inflammatory response and clinical worsening. Corticosteroids alone are preferred in such cases. Cysticidal therapy has no effect on calcified lesions.

\section{Role of surgery}

There has been a paradigm shift in the management practice of NCC, and currently there is no role of major surgical procedures except for some cases with extraparenchymal NCC. Surgical intervention such as endoscopic removal may be required in intraventricular and subarachnoid NCC, ventriculoperitoneal shunting for hydrocephalus, and excision of giant symptomatic cysts that fail to respond to medical therapy or intraocular cysts. ${ }^{90,91}$ Corticosteroids and albendazole are used simultaneously to reduce the rate of re-obstruction..$^{92}$

\section{Role of corticosteroids}

Oral corticosteroids are generally administered during the initiation of cysticidal therapy to reduce host inflammatory reaction. Pretreatment with corticosteroids also increases the plasma levels of albendazole sulfoxide. Intravenous dexamethasone $(0.1 / \mathrm{mg} / \mathrm{kg} /$ day $)$ may be used in cases with raised intracranial pressure, and prolonged oral corticosteroids are needed in children with numerous disseminated lesions, extensive cerebral edema, or intraocular cysticercosis. ${ }^{37}$ Role of corticosteroids in the resolution of cysts remains controversial and data are conflicting, thus requiring further research. ${ }^{93-96}$ We conducted a randomized trial among 133 children with SSECTL and found that disappearance of lesions at 3-month follow-up was somewhat higher (62.9\%) in the group that received corticosteroid plus albendazole as compared with albendazole alone $(59.5 \%)$ or steroids alone $(52.6 \%)$; however, this was not statistically significant. Children in the corticosteroid-alone group had significantly more seizure recurrences while on AEDs. ${ }^{94}$ Hence, our usual practice is to start oral corticosteroids (prednisolone $2 \mathrm{mg} / \mathrm{kg}$ / day after meals) 2-3 days before starting albendazole, and continue it for the next 3-4 days. Children with moderate to severe edema are kept under observation for 24-48 hours after starting albendazole.

\section{Choice of antiepileptic drug and duration of therapy}

Seizures secondary to NCC usually respond well to first-line AEDs. ${ }^{85}$ It is recommended to initiate monotherapy with a first-line drug and monitor for side effects as treating any case of seizures with an organic focus. ${ }^{97}$ Due to low cost and easy availability, carbamazepine and phenytoin are the usual choices in NCC-endemic areas. ${ }^{98,99}$ The optimal duration of AED therapy has been controversial, and a randomized study in 106 children with SSECTL showed that seizure recurrence after 1-year versus 2-year AED therapy was not 
significantly different. ${ }^{100} \mathrm{AED}$ can be withdrawn after 1-year seizure-free interval if the lesion has disappeared and the electroencephalograph has normalized. Seizure recurrence is low in children (10\%-20\% of cases), ${ }^{101,102}$ and correlates significantly with an abnormal CT (persistence or calcification of lesion or multiple lesions prior to starting cysticidal therapy) and an abnormal electroencephalograph at the time of AED withdrawal, ${ }^{100,103}$ thus requiring longer durations of AED. Side effects of the drugs such as Stevens-Johnson syndrome or other allergic reactions may warrant change of the AED.

\section{Prognosis of patients with NCC}

The follow-up of children with NCC needs to be individualized. A CT scan is usually done after 3-6 months to see whether the lesions have resolved. ${ }^{43}$ In children with persistent lesions, another course of cysticidal therapy is usually given. The outcome depends upon the type of NCC, cyst location (parenchymal better than extraparenchymal), and numbers (single lesions better than multiple). Single enhancing lesion NCC has a good prognosis - the lesion disappears within 6 months in more than $60 \%$ of the cases and seizures are well controlled. Recurrence of seizures in children with single lesions varies from $10 \%$ to $20 \%$, whereas multiple and calcified lesions have frequent seizure recurrences. The prognosis is poorer in cysticercus encephalitis and extraparenchymal NCC.

\section{Challenges in prevention}

NCC is a disease perpetuated by poor hygiene and sanitation and is entirely preventable and potentially eradicable. Risk factors determining the transmission of T. solium suggest that consumption of raw meat, inadequate or absence of meat inspection and control, poor sanitation, and use of untreated human waste as a fertilizer for crops may play important roles in endemic areas. ${ }^{104}$

Targeting the tapeworm carriers needs to be emphasized. Preventive measures include avoidance of open defecation, improved food-handling practices such as thorough cleaning of raw vegetables prior to use, wearing of gloves by food handlers, and mass administration of anthelminthic drugs in endemic areas. ${ }^{105,106}$ However, elimination of the parasite from the Southeast Asian regions has not been completely successful because of lack of integrated surveillance in both animals and human populations, nonavailability of tools for morphological or molecular identification of infection as $T$. solium, poor hygiene and sanitation standards, and persisting significant carrier population that can release thousands of eggs into the environment and also result in autoinfection. ${ }^{104,107}$ In certain Indonesian endemic regions, nearly $30 \%$ families have no sanitary facilities and people defecate in the garden, $11 \%$ pig owners keep their pigs in an open common pasture, and $2.2 \%$ even allow their pigs to roam free. ${ }^{108,109}$

Interruption of intestinal tapeworm transmission has mainly been based on eradication of swine cysticercosis through improved animal husbandry, inspection of pig carcasses in slaughterhouses, and meat inspection. However, ensuring universal meat inspection and good animal husbandry practices is easier said than done. Pig husbandry is still very traditional in certain regions of the world. Human defecation occurs quite often near the pigsties facilitating coprophagia by pigs. Slaughtering of pigs commonly occurs at home or in marketplaces with little veterinary supervision, and crude cysticercal detection methods such as tongue inspection in pigs are used. ${ }^{110,111}$

Ideal intervention programs to break the transmission cycle should include "one-health approach" targeting both humans and pigs within the same environment. Studies from hyperendemic areas show that after "one health intervention (triple dose of albendazole $400 \mathrm{mg}$ in the form of two mass drug administrations)" covering all village residents $>6$ years of age, the level of teniasis decreased by $79.4 \%$ after first regimen, remained steady during the 5-month inter-treatment interval and decreased again by $100 \%$ after second mass treatment regime. ${ }^{112} \mathrm{~A}$ prospective, interventional, cohort controlled study using the "ring-strategy" of screening and treatment for teniasis among households located within $100 \mathrm{~m}$ of pigs heavily infected with cysticercosis observed a $41 \%$ reduction in seroincidence and four times reduction in prevalence of NCC in the intervention village as compared to control village in northern Peru. ${ }^{113}$

No single effective vaccine for NCC is available till now. The complex immunology of the parasite, occult nature, and low morbidity of this infection make teniasis a poor candidate for vaccine development in humans. However, vaccinating pigs to prevent porcine cysticercosis is a good strategy. Recombinant oncosphere antigens TSOL18 and TSOL451A have been found to induce $100 \%$ protection in three independent vaccine trials in pigs in Mexico and Cameroon. ${ }^{114,115}$ Mass deworming of population with niclosamide or praziquantel and mass vaccination of pigs with TSOL16 and TSOL18 along with treatment of pigs with oxfendazole has been shown to be beneficial. ${ }^{116,117}$

For effective planning and implementation of prevention programs, it is essential to target public awareness regarding the disease. There are limited studies on the knowledge, attitude, and practice toward NCC. Hospital-, school-, and 
community-based studies from developing countries indicate low levels of awareness of teniasis and NCC amongst the hospital visitors, teachers, and general population. ${ }^{118-120}$

School children may be good targets for health education interventions as they may act as carriers of knowledge to adults and their communities, and thus may provide "herd-education" akin to herd immunity. Randomized studies from endemic areas report significant improvements in school children's knowledge regarding teniasis and NCC following health education intervention. ${ }^{121-123}$ Another thrust area of education intervention is the population of farmers dealing with pigs. A recent study from Kenya showed that workshops followed by one-on-one on-farm training enhanced the farmers' knowledge and awareness of the life cycle of $T$. solium and the transmission of NCC. ${ }^{124}$ The Salama-Honduras Study showed that communitybased interventions such as health education, animal husbandry training for pig farmers, construction of water projects and proper sewage disposal, construction of maternal and child health clinic, mass deworming of school students, ongoing teniasis surveillance, and carrier detection helped reduce preventable epilepsy from NCC in the hyperendemic population even in resource-constrained developing country. ${ }^{15}$

Finally, a multipronged action plan that includes public education, proper hygiene, and sanitation; encouraging pig farmers to keep their pigs penned or tethered to prevent them from scavenging; encouraging people living in endemic areas to cook meat thoroughly; observing strict hygiene and avoid defecating in the open; enforcing strict animal husbandry and meat inspection procedures are warranted.

\section{Future prospects}

In order to improve the knowledge of NCC, it is important to develop validated criteria for diagnosis, since the current available diagnostic criteria have not been validated so far. Newer insights into the immune mechanisms underlying symptomatic human cysticercosis and helminthinduced immune suppression are being obtained through recent studies. Toll-like receptor-4 and soluble intercellular adhesion molecule1 K469E polymorphisms have been suggested to predispose to symptomatic infection. ${ }^{125,126}$ The role of genetic susceptibility to NCC is also being evaluated and preliminary work has reported positive association of HLADRBII-13 with SSECTL(s). ${ }^{127}$ Prospective cohort studies about epileptogenicity of NCC are needed to assess the association of different evolutionary phases of the parasite, in the development of seizures and epilepsy. ${ }^{128}$

The understanding of these immune and genetic mechanisms will help develop newer drugs such as tamoxifen and newer drug delivery systems such as lactic acid conjugated solid lipid nanoparticles bearing albendazole and prednisolone, for effective management of NCC. ${ }^{129,130}$ Results of preclinical animal studies on the pharmacokinetics, safety, and toxicology of oxfendazole for humans have been encouraging. ${ }^{131}$ Development of effective antiparasitic drugs for treatment of swine cysticercosis is also a potential area of research, especially combination therapy. ${ }^{97}$

\section{Conclusion}

NCC is an important acquired cause of epilepsy and other neurological manifestations especially in endemic areas. Because of its pleomorphic presentation, NCC should be considered in the differential diagnosis of a number of neurological conditions. Treatment with cysticidal therapy leads to reduction in seizure frequency and a faster resolution of lesions. A single AED, usually carbamazepine or phenytoin, is sufficient to control seizures due to singlelesion NCC. Cases with single or few lesions have a good outcome. Prevention of NCC is important and is feasible. Public sanitation and hygiene awareness are of utmost importance to prevention of this disease. Development of newer cysticidal drugs and drug delivery systems for both human and swine population are the potential areas of research.

\section{Acknowledgment}

This study did not receive funding from any organization.

\section{Disclosure}

The authors report no conflicts of interest in this work.

\section{References}

1. Epilepsy CoTDotILA. Relationship between epilepsy and tropical diseases. Epilepsia. 1994;35:89-93.

2. Singhi P, Ray M, Singhi S, and Khandelwal N. Clinical spectrum of 500 children with neurocysticercosis and response to albendazole therapy. J Child Neurol. 2000;15:207-213.

3. WHO. Accelerating Work to Overcome the Global Impact of Neglectd Tropical Diseases. A roadmap for Impementation. Geneva: World Health Organization; 2012

4. Del Brutto OH, Garcia HH. Neurocysticercosis in nonendemic countries: time for a reappraisal. Neuroepidemiology. 2012;39:145-146.

5. Serpa JA, White AC Jr. Neurocysticercosis in the United States. Pathog Glob Health. 2012;106:256-260.

6. Zammarchi L, Strohmeyer M, Bartalesi F, et al. Epidemiology and management of cysticercosis and Taenia solium taeniasis in Europe, systematic review 1990-2011. PLoS One. 2013;8:e69537.

7. Cherian A, Syam UK, Sreevidya D, et al. Low seroprevalence of systemic cysticercosis among patients with epilepsy in Kerala - South India. J Infect Public Health. 2014;7:271-276.

8. Mafojane NA, Appleton CC, Krecek RC, Michael LM, Willingham AL 3rd. The current status of neurocysticercosis in Eastern and Southern Africa. Acta Trop. 2003;87:25-33.

9. Montano SM, Villaran MV, Ylquimiche L, et al. Neurocysticercosis: association between seizures, serology, and brain CT in rural Peru. Neurology. 2005;65:229-233. 
10. Parija SC, Balamurungan N, Sahu PS, Subbaiah SP. Cysticercus antibodies and antigens in serum from blood donors from Pondicherry, India. Rev Inst Med Trop Sao Paulo. 2005;47:227-230.

11. Prasad KN, Prasad A, Gupta RK, et al. Neurocysticercosis in patients with active epilepsy from the pig farming community of Lucknow district, north India. Trans R Soc Trop Med Hyg. 2009;103: 144-150.

12. Sahu PS, Patro S, Jena PK, Swain SK, Das BK. Imaging and serologicalevidence of neurocysticercosis among patients with seizures in Odisha, an unexplored Eastern Coastal Province in India. J Clin Diagn Res. 2015;9:DC06-DC10.

13. Khurana S, Aggarwal A, Malla N. Prevalence of anti-cysticercus antibodies in slum, rural and urban populations in and around Union territory, Chandigarh. Indian J Pathol Microbiol. 2006;49:51-53.

14. Prasad KN, Verma A, Srivastava S, et al. An epidemiological study of asymptomatic neurocysticercosis in a pig farming community in northern India. Trans R Soc Trop Med Hyg. 2011;105:531-536.

15. Medina MT, Aguilar-Estrada RL, Alvarez A, et al. Reduction in rate of epilepsy from neurocysticercosis by community interventions: the Salama, Honduras study. Epilepsia. 2011;52:1177-1185.

16. Ratnam S, Khanna PN, and Bandyopadhyay AK. Incidence of taeniasis in man. Indian J Public Health. 1983;27:70-74.

17. Prasad KN, Chawla S, Jain D, et al. Human and porcine Taenia solium infection in rural north India. Trans R Soc Trop Med Hyg. 2002;96: 515-516.

18. Nash TE, Mahanty S, Garcia HH. Neurocysticercosis-more than a neglected disease. PLoS Negl Trop Dis. 2013;7:e1964.

19. Ndimubanzi PC, Carabin H, Budke CM, et al. A systematic review of the frequency of neurocyticercosis with a focus on people with epilepsy. PLoS Negl Trop Dis. 2010;4:e870.

20. Quet F, Guerchet M, Pion SD, et al. Meta-analysis of the association between cysticercosis and epilepsy in Africa. Epilepsia. 2010;51: 830-837.

21. Singh G, Bawa J, Chinna D, et al. Association between epilepsy and cysticercosis and toxocariasis: a population-based case-control study in a slum in India. Epilepsia. 2012;53:2203-2208.

22. Raina SK, Razdan S, Pandita KK, Sharma R, Gupta VP. Active epilepsy as indicator of neurocysticercosis in rural northwest India. Epilepsy Res Treat. 2012;2012:802747.

23. Singhi P, Singhi S. Neurocysticercosis in children. J Child Neurol. 2004;19:482-492.

24. Ito A, Putra MI, Subahar R, et al. Dogs as alternative intermediate hosts of Taenia solium in Papua (Irian Jaya), Indonesia confirmed by highly specific ELISA and immunoblot using native and recombinant antigens and mitochondrial DNA analysis. J Helminthol. 2002;76:311-314.

25. Schantz PM, Moore AC, Munoz JL, et al. Neurocysticercosis in an orthodox Jewish community in New York City. $N$ Engl J Med. 1992;327:692-695.

26. Del Brutto OH. Neurocysticercosis: new thoughts on controversial issues. Curr Opin Neurol. 2013;26:289-294.

27. Del Brutto OH, Campos X. Massive neurocysticercosis: encephalitic versus non-encephalitic. Am J Trop Med Hyg. 2012;87:381.

28. Escobar A. The pathology of neurocysticercosis. In, Palacios E, Rodriguez-Carbajal J, Taveras JM, editors. Cysticercosis of Central Nervous System. Springfield, IL, USA: Charles C Thomas. 1983;27-54.

29. Nash TE, Pretell EJ, Lescano AG, et al. Perilesional brain oedema and seizure activity in patients with calcified neurocysticercosis: a prospective cohort and nested case-control study. Lancet Neurol. 2008;7:1099-1105.

30. Carpio A, Romo ML. The relationship between neurocysticercosis and epilepsy: an endless debate. Arq Neuropsiquiatr. 2014;72:383-390.

31. Rathore C, Thomas B, Kesavadas C, Radhakrishnan K. Calcified neurocysticercosis lesions and hippocampal sclerosis: potential dual pathology? Epilepsia. 2012;53:e60-e62.

32. Gupta RK, Awasthi R, Rathore RK, et al. Understanding epileptogenesis in calcified neurocysticercosis with perfusion MRI. Neurology. 2012;78:618-625.
33. Del Brutto OH, Del Brutto VJ. Calcified neurocysticercosis among patients with primary headache. Cephalalgia. 2012;32:250-254.

34. Ito A, Wandra $\mathrm{T}$, $\mathrm{Li} \mathrm{T}$, et al. The present situation of human taeniases and cysticercosis in Asia. Recent Pat Antiinfect Drug Discov. 2014;9: 173-185.

35. Del Brutto OH, Wadia NH, Dumas M, et al. Proposal of diagnostic criteria for human cysticercosis and neurocysticercosis. J Neurol Sci. 1996;142:1-6.

36. Del Brutto OH. Diagnostic criteria for neurocysticercosis, revisited. Pathog Glob Health. 2012;106:299-304

37. Singhi P, Singhi S. Neurocysticercosis in children. Indian J Pediatr. 2009;76:537-545

38. Singhi P, Saini AG, Sankhyan N. Amaurosis fugax caused by neurocysticercosis. Pediatr Infect Dis J. 2014;33:427.

39. Zhao J-L, Lerner A, Shu Z, Gao X-J, Zee C-S. Imaging spectrum of neurocysticercosis. Radiology of Infectious Diseases. 2015;1:94-102.

40. Kimura-Hayama ET, Higuera JA, Corona-Cedillo R, et al. Neurocysticercosis: radiologic-pathologic correlation. Radiographics. 2010;30:1705-1719.

41. Garcia $\mathrm{HH}$, Del Brutto $\mathrm{OH}$. Imaging findings in neurocysticercosis. Acta Trop. 2003;87:71-78.

42. Singhi PD, Baranwal AK. Single small enhancing computed tomographic lesion in Indian children - I: evolution of current concepts. J Trop Pediatr. 2001;47:204-207.

43. Singhi PD, Baranwal AK. Single small enhancing computed tomographic lesions in indian children - II. Clinical features, pathology, radiology and management. J Trop Pediatr. 2001;47:266-270.

44. Pretell EJ, Martinot C Jr, Garcia HH, et al. Differential diagnosis between cerebral tuberculosis and neurocysticercosis by magnetic resonance spectroscopy. J Comput Assist Tomogr. 2005;29:112-114.

45. Rajshekhar V, Haran RP, Prakash GS, Chandy MJ. Differentiating solitary small cysticercus granulomas and tuberculomas in patients with epilepsy. Clinical and computerized tomographic criteria. J Neurosurg. 1993;78:402-407.

46. Govindappa SS, Narayanan JP, Krishnamoorthy VM, et al. Improved detection of intraventricular cysticercal cysts with the use of threedimensional constructive interference in steady state MR sequences. AJNR Am J Neuroradiol. 2000;21:679-684.

47. Gupta RK, Prakash M, Mishra AM, et al. Role of diffusion weighted imaging in differentiation of intracranial tuberculoma and tuberculous abscess from cysticercus granulomas-a report of more than 100 lesions. Eur J Radiol. 2005;55:384-392.

48. de Souza A, Nalini A, Kovoor JM, et al. Perilesional gliosis around solitary cerebral parenchymal cysticerci and long-term seizure outcome: a prospective study using serial magnetization transfer imaging. Epilepsia. 2011;52:1918-1927.

49. do Amaral LL, Ferreira RM, da Rocha AJ, Ferreira NP. Neurocysticercosis: evaluation with advanced magnetic resonance techniques and atypical forms. Top Magn Reson Imaging. 2005;16:127-144.

50. Carrillo Mezo R, Lara Garcia J, Arroyo M, Fleury A. Relevance of 3D magnetic resonance imaging sequences in diagnosing basal subarachnoid neurocysticercosis. Acta Trop. 2015;152:60-65.

51. Atluri SR, Singhi P, Khandelwal N, Malla N. Evaluation of excretory secretory and 10-30 kDa antigens of Taenia solium cysticerci by EITB assay for the diagnosis of neurocysticercosis. Parasite Immunol. 2009;31:151-155

52. Sahu PS, Parija SC, Jayachandran S. Antibody specific to $43 \mathrm{kDa}$ excretory-secretory antigenic peptide of Taenia solium metacestode as a potential diagnostic marker in human neurocysticercosis. Acta Trop. 2010;115:257-261.

53. Atluri SR, Singhi P, Khandelwal N, Malla N. Neurocysticercosis immunodiagnosis using Taenia solium cysticerci crude soluble extract, excretory secretory and lower molecular mass antigens in serum and urine samples of Indian children. Acta Trop. 2009;110:22-27.

54. Sahu PS, Parija S, Kumar D, Jayachandran S, Narayan S. Comparative profile of circulating antigenic peptides in CSF, serum and urine from patients with neurocysticercosis diagnosed by immunoblotting. Parasite Immunol. 2014;36:509-521. 
55. Atluri VS, Gogulamudi VR, Singhi P, et al. Pediatric neurocysticercosis: usefulness of antibody response in cysticidal treatment follow-up. Biomed Res Int. 2014;2014:904046.

56. Ito A, Yamasaki H, Nakao M, et al. Multiple genotypes of Taenia solium - ramifications for diagnosis, treatment and control. Acta Trop. 2003;87:95-101.

57. Wilson M, Bryan RT, Fried JA, et al. Clinical evaluation of the cysticercosis enzyme-linked immunoelectrotransfer blot in patients with neurocysticercosis. J Infect Dis. 1991;164:1007-1009.

58. Mandal J, Singhi PD, Khandelwal N, Malla N. Evaluation of ELISA and dot blots for the serodiagnosis of neurocysticercosis, in children found to have single or multiple enhancing lesions in computerized tomographic scans of the brain. Ann Trop Med Parasitol. 2006;100:39-48.

59. Rodriguez S, Dorny P, Tsang VC, et al. Detection of Taenia solium antigens and anti-T. solium antibodies in paired serum and cerebrospinal fluid samples from patients with intraparenchymal or extraparenchymal neurocysticercosis. J Infect Dis. 2009;199:1345-1352.

60. Noh J, Rodriguez S, Lee YM, et al. Recombinant protein- and synthetic peptide-based immunoblot test for diagnosis of neurocysticercosis J Clin Microbiol. 2014;52:1429-1434.

61. Garcia HH, Gilman RH, Catacora M, et al. Serologic evolution of neurocysticercosis patients after antiparasitic therapy. Cysticercosis Working Group in Peru. J Infect Dis. 1997;175:486-489.

62. Rodriguez-Canul R, Allan JC, Fletes C, et al. Comparative evaluation of purified Taenia solium glycoproteins and crude metacestode extracts by immunoblotting for the serodiagnosis of human T. solium cysticercosis. Clin Diagn Lab Immunol. 1997;4:579-582.

63. Mandal J, Singhi PD, Khandelwal N, Malla N. Evaluation of lower molecular mass (20-24 kDa) Taenia solium cysticercus antigen fraction by ELISA and dot blot for the serodiagnosis of neurocysticercosis in children. Parasitol Res. 2008;102:1097-1101.

64. Hell RC, Amim P, de Andrade HM, et al. Immunodiagnosis of human neurocysticercosis using a synthetic peptide selected by phage-display. Clin Immunol. 2009;131:129-138.

65. Ribeiro Vda S, Nunes Dda S, Gonzaga HT, da Cunha-Junior JP, Costa-Cruz JM. Diethylaminoethyl (DEAE) binding fraction from Taenia solium metacestode improves the neurocysticercosis serodiagnosis. Parasitol Res. 2014;113:2569-2575.

66. Handali S, Klarman M, Gaspard AN, et al. Development and evaluation of a magnetic immunochromatographic test to detect Taenia solium, which causes taeniasis and neurocysticercosis in humans. Clin Vaccine Immunol. 2010;17:631-637.

67. Corstjens PL, de Dood CJ, Priest JW, Tanke HJ, Handali S. Feasibility of a lateral flow test for neurocysticercosis using novel up-converting nanomaterials and a lightweight strip analyzer. PLoS Negl Trop Dis. 2014;8:e2944.

68. Michelet L, Fleury A, Sciutto E, et al. Human neurocysticercosis: comparison of different diagnostic tests using cerebrospinal fluid. J Clin Microbiol. 2011;49:195-200.

69. Yamasaki H, Allan JC, Sato MO, et al. DNA differential diagnosis of taeniasis and cysticercosis by multiplex PCR. J Clin Microbiol. 2004;42:548-553.

70. Yera H, Dupont D, Houze S, et al. Confirmation and follow-up of neurocysticercosis by real-time PCR in cerebrospinal fluid samples of patients living in France. J Clin Microbiol. 2011;49:4338-4340.

71. Camargo JA, Bertolucci PH. Quantification of Fas protein in CSF of patients with neurocysticercosis. Arq Neuropsiquiatr. 2012;70:262-263

72. Camargo JA, Bertolucci PH. Quantification of amino acid neurotransmitters in cerebrospinal fluid of patients with neurocysticercosis. Open Neurol J. 2015;9:15-20.

73. Singhi P. Neurocysticercosis. Ther Adv Neurol Disord. 2011;4:67-81.

74. Abba K, Ramaratnam S, Ranganathan LN. Anthelmintics for people with neurocysticercosis. Cochrane Database Syst Rev. 2010; CD000215.

75. Del Brutto OH, Roos KL, Coffey CS, Garcia HH. Meta-analysis: Cysticidal drugs for neurocysticercosis: albendazole and praziquantel. Ann Intern Med. 2006;145:43-51.
76. Mazumdar M, Pandharipande P, Poduri A. Does albendazole affect seizure remission and computed tomography response in children with neurocysticercosis? A Systematic review and meta-analysis. J Child Neurol. 2007;22:135-142.

77. Baranwal AK, Singhi PD, Khandelwal N, Singhi SC. Albendazole therapy in children with focal seizures and single small enhancing computerized tomographic lesions: a randomized, placebo-controlled, double blind trial. Pediatr Infect Dis J. 1998;17:696-700.

78. Kalra V, Dua T, Kumar V. Efficacy of albendazole and short-course dexamethasone treatment in children with 1 or 2 ring-enhancing lesions of neurocysticercosis: a randomized controlled trial. J Pediatr. 2003;143:111-114.

79. Thussu A, Chattopadhyay A, Sawhney IM, Khandelwal N. Albendazole therapy for single small enhancing CT lesions (SSECTL) in the brain in epilepsy. J Neurol Neurosurg Psychiatry. 2008;79:272-275.

80. Chaurasia RN, Garg RK, Agarwall A, et al. Three day albendazole therapy in patients with a solitary cysticercus granuloma: a randomized double blind placebo controlled study. Southeast Asian J Trop Med Public Health. 2010;41:517-525.

81. Otte WM, Singla M, Sander JW, Singh G. Drug therapy for solitary cysticercus granuloma: a systematic review and meta-analysis. Neurology. 2013;80:152-162.

82. Baird RA, Wiebe S, Zunt JR, et al. Evidence-based guideline: treatment of parenchymal neurocysticercosis: report of the Guideline Development Subcommittee of the American Academy of Neurology. Neurology. 2013;80:1424-1429.

83. Jung H, Hurtado M, Sanchez M, Medina MT, Sotelo J. Plasma and CSF levels of albendazole and praziquantel in patients with neurocysticercosis. Clin Neuropharmacol. 1990;13:559-564.

84. Jung H, Hurtado M, Medina MT, Sanchez M, Sotelo J. Dexamethasone increases plasma levels of albendazole. J Neurol. 1990;237:279-280.

85. Garcia HH, Evans CA, Nash TE, et al. Current consensus guidelines for treatment of neurocysticercosis. Clin Microbiol Rev. 2002;15: 747-756.

86. Singhi P, Dayal D, Khandelwal N. One week versus four weeks of albendazole therapy for neurocysticercosis in children: a randomized, placebo-controlled double blind trial. Pediatr Infect Dis J. 2003;22: 268-272.

87. Pretell EJ, Garcia HH, Gilman RH, Saavedra H, Martinez M. Failure of one-day praziquantel treatment in patients with multiple neurocysticercosis lesions. Clin Neurol Neurosurg. 2001;103:175-177.

88. Kaur S, Singhi P, Singhi S, Khandelwal N. Combination therapy with albendazole and praziquantel versus albendazole alone in children with seizures and single lesion neurocysticercosis: a randomized, placebocontrolled double blind trial. Pediatr Infect Dis J. 2009;28:403-406.

89. Garcia HH, Gonzales I, Lescano AG, et al. Efficacy of combined antiparasitic therapy with praziquantel and albendazole for neurocysticercosis: a double-blind, randomised controlled trial. Lancet Infect Dis. 2014;14:687-695.

90. Goel RK, Ahmad FU, Vellimana AK, et al. Endoscopic management of intraventricular neurocysticercosis. J Clin Neurosci. 2008;15: 1096-1101.

91. Suri A, Goel RK, Ahmad FU, et al. Endoscopic excision of intraventricular neurocysticercosis in children: a series of six cases and review. Childs Nerv Syst. 2008;24:281-285.

92. Khade P, Lemos RS, Toussaint LG. What is the utility of postoperative antihelminthic therapy after resection for intraventricular neurocysticercosis? World Neurosurg. 2013;79:558-567.

93. Carpio A, Santillan F, Leon P, Flores C, Hauser WA. Is the course of neurocysticercosis modified by treatment with antihelminthic agents? Arch Intern Med. 1995;155:1982-1988.

94. Singhi P, Jain V, Khandelwal N. Corticosteroids versus albendazole for treatment of single small enhancing computed tomographic lesions in children with neurocysticercosis. J Child Neurol. 2004;19:323-327.

95. Kishore D, Misra S. Short course of oral prednisolone on disappearance of lesion and seizure recurrence in patients of solitary cysticercal granuloma with single small enhancing CT lesion: an open label randomized prospective study. J Assoc Physicians India. 2007;55:419-424. 
96. Mall RK, Agarwal A, Garg RK, Kar AM, Shukla R. Short course of prednisolone in Indian patients with solitary cysticercus granuloma and new-onset seizures. Epilepsia. 2003;44:1397-1401.

97. Gonzales I, Garcia HH. Current status and future perspectives on the medical treatment of neurocysticercosis. Pathog Glob Health. 2012;106:305-309.

98. Nash TE, Garcia HH. Diagnosis and treatment of neurocysticercosis. Nat Rev Neurol. 2011;7:584-594.

99. Garcia HH, Gonzalez AE, Gilman RH. Cysticercosis of the central nervous system: how should it be managed? Curr Opin Infect Dis. 2011;24:423-427.

100. Singhi PD, Dinakaran J, Khandelwal N, Singhi SC. One vs two years of anti-epileptic therapy in children with single small enhancing CT lesions. J Trop Pediatr. 2003;49:274-278.

101. Talukdar B, Saxena A, Popli VK, Choudhury V. Neurocysticercosis in children: clinical characteristics and outcome. Ann Trop Paediatr. 2002;22:333-339.

102. Baranwal AK, Singhi PD, Singhi SC, Khandelwal N. Seizure recurrence in children with focal seizures and single small enhancing computed tomographic lesions: prognostic factors on long-term follow-up. J Child Neurol. 2001;16:443-445.

103. Goel D, Mittal M, Bansal KK, Singhal A. Natural history of solitary cerebral cysticercosis cases after albendazole therapy: a longitudinal follow-up study from India. Acta Neurol Scand. 2010;121:204-208.

104. Van De N, Le TH, Lien PT, Eom KS. Current status of taeniasis and cysticercosis in Vietnam. Korean J Parasitol. 2014;52:125-129.

105. Garcia HH, Del Brutto OH, Nash TE, et al. New concepts in the diagnosis and management of neurocysticercosis (Taenia solium). Am J Trop Med Hyg. 2005;72:3-9.

106. Lightowlers MW, Colebrook AL, Gauci CG, et al. Vaccination against cestode parasites: anti-helminth vaccines that work and why. Vet Parasitol. 2003;115:83-123.

107. Willingham AL 3rd, Wu HW, Conlan J, Satrija F. Combating Taenia solium cysticercosis in Southeast Asia an opportunity for improving human health and livestock production. Adv Parasitol. 2010;72:235-266

108. Wandra T, Swastika K, Dharmawan NS, et al. The present situation and towards the prevention and control of neurocysticercosis on the tropical island, Bali, Indonesia. Parasit Vectors. 2015;8:148.

109. Wandra T, Ito A, Swastika K, et al. Taeniases and cysticercosis in Indonesia: past and present situations. Parasitology. 2013;140:1608-1616.

110. Pouedet MS, Zoli AP, Nguekam, et al. Epidemiological survey of swine cysticercosis in two rural communities of West-Cameroon. Vet Parasitol. 2002;106:45-54.

111. Shey-Njila O, Zoli PA, Awah-Ndukum J, et al. Porcine cysticercosis in village pigs of North-West Cameroon. J Helminthol. 2003;77:351-354.

112. Ash A, Okello A, Khamlome B, et al. Controlling Taenia solium and soil transmitted helminths in a northern Lao PDR village: impact of a triple dose albendazole regime. Acta Trop. 2015.

113. O’Neal SE, Moyano LM, Ayvar V, et al. Ring-screening to control endemic transmission of Taenia solium. PLoS Negl Trop Dis. 2014;8:e3125.

114. Flisser A, Gauci CG, Zoli A, et al. Induction of protection against porcine cysticercosis by vaccination with recombinant oncosphere antigens. Infect Immun. 2004;72:5292-5297.

Pediatric Health, Medicine and Therapeutics

\section{Publish your work in this journal}

Pediatric Health, Medicine and Therapeutics is an international, peerreviewed, open access journal publishing original research, reports, editorials, reviews and commentaries. All aspects of health maintenance, preventative measures and disease treatment interventions are addressed within the journal. Practitioners from all disciplines are invited to submit
115. Lightowlers MW. Vaccination for the prevention of cysticercosis. Dev Biol (Basel). 2004;119:361-368.

116. Jayashi CM, Kyngdon CT, Gauci CG, Gonzalez AE, Lightowlers MW. Successful immunization of naturally reared pigs against porcine cysticercosis with a recombinant oncosphere antigen vaccine. Vet Parasitol. 2012;188:261-267.

117. Sikasunge CS, Johansen MV, Phiri IK, Willingham AL 3rd, Leifsson PS. The immune response in Taenia solium neurocysticercosis in pigs is associated with astrogliosis, axonal degeneration and altered bloodbrain barrier permeability. Vet Parasitol. 2009;160:242-250.

118. Girotra M, Gera C, Abraham RR, et al. Awareness of neurocysticercosis: a study from northwest India. Ann Indian Acad Neurol. 2011;14:27-30.

119. Mishra D, Kalra V, Aggarwal K. Awareness about taeniasis and neurocysticercosis among municipal schoolteachers in Delhi. J Commun Dis. 2007;39:225-228.

120. Sarti E, Flisser A, Schantz PM, et al. Development and evaluation of a health education intervention against Taenia solium in a rural community in Mexico. Am J Trop Med Hyg. 1997;56:127-132.

121. Mwidunda SA, Carabin H, Matuja WB, Winkler AS, Ngowi HA. A school based cluster randomised health education intervention trial for improving knowledge and attitudes related to Taenia solium cysticercosis and taeniasis in Mbulu district, northern Tanzania. PLoS One. 2015;10:e118541.

122. Alexander AM, Mohan VR, Muliyil J, Dorny P, Rajshekhar V. Changes in knowledge and practices related to taeniasis/cysticercosis after health education in a south Indian community. Int Health. 2012;4: 164-169.

123. Veena K, Devendra M, and Kiran A. School-based health education intervention for prevention of taeniasis and neurocysticercosis: a pilot study. J Commun Dis. 2012;44:223-230.

124. Wohlgemut J, Dewey C, Levy M, Mutua F. Evaluating the efficacy of teaching methods regarding prevention of human epilepsy caused by Taenia solium neurocysticercosis in Western Kenya. Am J Trop Med Hyg. 2010;82:634-642.

125. Verma A, Prasad KN, Gupta RK, et al. Toll-like receptor 4 polymorphism and its association with symptomatic neurocysticercosis. J Infect Dis. 2010;202:1219-1225.

126. Singh A, Singh AK, Singh SK, et al. Association of ICAM-1 K469E polymorphism with neurocysticercosis. J Neuroimmunol. 2014;276: 166-171.

127. Jain S, Padma MV, Kanga U, et al. Family studies and human leukocyte antigen class II typing in Indian probands with seizures in association with single small enhancing computed tomography lesions. Epilepsia. 1999;40:232-238.

128. Carpio A, Romo ML. Multifactorial basis of epilepsy in patients with neurocysticercosis. Epilepsia. 2015;56:973-974.

129. Panic G, Duthaler U, Speich B, Keiser J. Repurposing drugs for the treatment and control of helminth infections. Int J Parasitol Drugs Drug Resist. 2014;4:185-200.

130. Devi R, Jain A, Hurkat P, Jain SK. Dual drug delivery using lactic acid conjugated SLN for effective management of neurocysticercosis. Pharm Res. 2015;32(10):3137-3148.

131. Codd EE, Ng HH, McFarlane C, et al. Preclinical studies on the pharmacokinetics, safety, and toxicology of oxfendazole: toward first in human studies. Int J Toxicol. 2015;34:129-137.

\section{Dovepress}

their work as well as healthcare researchers and patient support groups. The manuscript management system is completely online and includes a very quick and fair peer-review system. Visit http://www.dovepress.com/ testimonials.php to read real quotes from published authors. 